\title{
Domain walls in noncommutative gauge theories, folded D-branes, and communication with mirror world
}

\author{
S.L. Dubovsky ${ }^{a, b}$, S.M. Sibiryakov ${ }^{b}$ \\ ${ }^{a}$ Department of Physics, CERN Theory Division CH-1211 Geneva 23, Switzerland \\ ${ }^{b}$ Institute for Nuclear Research of the Russian Academy of Sciences, \\ 60th October Anniversary Prospect, 7a, 117312 Moscow, Russia
}

\begin{abstract}
Noncommutative $U(\mathcal{N})$ gauge theories at different $\mathcal{N}$ may be often thought of as different sectors of a single theory. For instance, $U(1)$ theory possesses a sequence of vacua labeled by an integer parameter $\mathcal{N}$, and the theory in the vicinity of the $\mathcal{N}$-th vacuum coincides with the $U(\mathcal{N})$ noncommutative gauge theory. We construct domain walls on noncommutative plane, which separate vacua with different gauge groups in gauge theory with adjoint scalar field. The scalar field has nonminimal coupling to the gauge field, such that the scale of noncommutativity is determined by the vacuum value of the scalar field. The domain walls are solutions of the BPS equations in the theory. It is natural to interprete the domain wall as a stack of D-branes plus a stack of folded D-branes. We support this interpretation by the analysis of small fluctuations around domain walls, and suggest that such configurations of branes emerge as solutions of the Matrix model in large class of pp-wave backgrounds with inhomogeneous field strength. We point out that the folded D-brane per se provides an explicit realization of the "mirror world" idea, and speculate on some phenomenological consequences of this scenario.
\end{abstract}

\section{Introduction}

Recent interest in noncommutative (NC) gauge theories is to large extent motivated by rich pattern of stringy phenomena exhibited in these theories. One of the spectacular manifestations of the stringy origin of $\mathrm{NC}$ gauge theories is that in many cases $[1,2,3,4]$ the rank $\mathcal{N}$ of gauge group is a background dependent parameter. For instance, it was pointed out in Ref. [1] that $U(1)$ gauge theory on NC plane possesses a sequence of vacua labeled by a natural number $\mathcal{N}$ with the following peculiar properties:

i) Every vacuum with $\mathcal{N}>1$ is a highly non-local field configuration from the point of view of the trivial $(\mathcal{N}=1)$ vacuum; 
ii) Perturbation theory in the vicinity of the $\mathcal{N}$-th vacuum is equivalent to perturbation theory of the $U(\mathcal{N})$ NC gauge theory above its trivial vacuum;

iii) The fact that there are different gauge theories in different vacua cannot be understood as Higgs mechanism. Namely, the action in the vicinity of the $\mathcal{N}$-th vacuum contains $U(\mathcal{N})$ gauge fields only, with no extra massive vector bosons or Higgs fields.

A possible interpretation of this phenomenon is that $U(\mathcal{N})$ NC gauge theory "remembers" its origin in string theory, where it emerges as the effective field theory describing a stack of $\mathcal{N}$ D-branes with constant $B$-field in the zero slope limit [5] and, thus, the rank of gauge group $\mathcal{N}$ is a parameter of the background. However, in the case of pure gauge theory on the $\mathrm{NC}$ plane it was argued [1] that $\mathcal{N}$ is a superselection parameter, implying that different sectors are completely disconnected from each other. Were this the whole story, one could object that in this respect NC theories are not more "stringy" than ordinary Yang-Mills theories. Indeed, one may think that the very existence of ordinary $U(\mathcal{N})$ theories with different $\mathcal{N}$ is also a reflection of the fact that ordinary gauge theory appears as the low energy description of a stack of $\mathcal{N}$ D-branes in string theory.

However, recently it was demonstrated that in some NC theories, it is possible to construct domain walls [6] (see also Ref. [7] for earlier related work) interpolating between vacua with different gauge groups. Consequently, the rank of the gauge group can be a nontrivial dynamical parameter in $\mathrm{NC}$ gauge theories. This makes the above phenomenon significantly more interesting from the viewpoint of both field and string theory.

The NC manifold considered in Ref. [6] is fuzzy culinder. Although the fuzzy cylinder setup enables one to construct domain walls interpolating between vacua with $U(\mathcal{N})$ and $U(\mathcal{M})$ gauge groups for arbitrary $\mathcal{N}$ and $\mathcal{M}$, it has a number of drawbacks. First, the algebra of functions on fuzzy cylinder is different from that on the NC plane. As a result, some formulae become more complicated and obscure. Another, less technical problem is that fuzzy cylinder can be interpreted as a cylindrical semi-lattice. This may give rise to a suspicion, that it is the lattice structure which is crucial for the existence of the domain walls, and that they are absent in a continuous setting. This suspicion is further corroborated by the fact that the tension of walls constructed in Ref. [6] diverges in the limit when the fuzzy cylinder approaches NC plane.

The main purpose of this paper is to demonstrate that domain walls between vacua with different gauge groups exist also in theories on the NC plane without any lattice structure. According to Ref. [1], these theories cannot be pure Yang-Mills. To understand the ingredients which should be added, it is instructive to think about possible string theoretical 


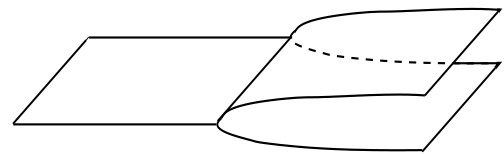

a

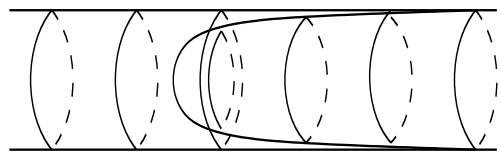

$\mathrm{b}$

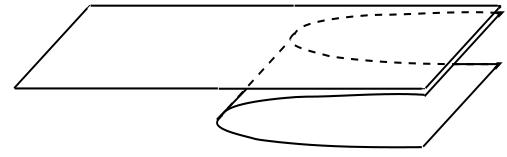

C

Figure 1: a) hypothetical junction of D-branes, b) geometrical interpretation of a domain wall on the fuzzy cylinder, c) geometrical interpretation of a domain wall on NC plane.

interpretation of the domain walls. The first idea may be that the domain wall is a kind of a junction of D-branes. For instance, in Fig. 1a, we show the hypothetical junction which would correspond to a domain wall between $U(1)$ and $U(2)$ vacua. However, junctions of this type do not exist in string theory. The geometrical interpretation of the domain walls on the fuzzy cylinder suggests a different picture. Namely, these domain walls can be understood as a set of infinite cylindrical branes and half-infinite branes of fingerstall shape [6]. In Fig. 1b the brane configuration corresponding to $U(1)-U(2)$ domain wall on the fuzzy cylinder is shown. A natural analogue of this configuration in the planar case is shown in Fig. 1c. In the rest of the paper we describe domain walls on NC plane and demonstrate that their properties match the ones expected for the D-brane configuration of Fig. 1c.

The first ingredient needed to construct a realization of the brane configuration of Fig. 1c in $\mathrm{NC}$ gauge theory is extra scalar field $\phi$ in the adjoint representation of the gauge group. This field corresponds to the transverse coordinate in Fig. 1c and parametrizes the relative positions of D-branes in the transverse direction. Scalar fields of this type are generic in gauge theories residing on stacks of D-branes both in commutative and noncommutative cases.

Another modification of pure NC Yang-Mills theory needed for incorporating domain walls is less trivial. It stems from the fact that the two sheets of the folded brane in Fig. 1c appear as a brane-antibrane pair far from the tip of the fold (and not as a pair of two Dbranes). A simple way to understand this is to consider the folded brane as the limiting configuration obtained from the cylindrical brane by elliptic deformation (c.f. Refs. [8, 10]). As the cylindrical brane carries no net RR-charge, two sheets of the fold should carry opposite charges. To figure out what is needed to satisfy this requirement in the $\mathrm{NC}$ theory, it is helpful to use the language of the BFSS Matrix model [11]. There, the RR-charge density in 
the transverse plane $(X, Y)$ is proportional to the commutator of the corresponding matrix coordinates,

$$
\rho_{X Y} \propto i[X, Y]
$$

and this charge density should take opposite values on the two sheets of the folded brane. On the other hand, in the NC theory the commutator of two coordinates is the parameter of the noncommutativity,

$$
[X, Y]=i \theta
$$

Consequently, one should allow the parameter of noncommutativity to vary in space, so that it takes opposite values at the two sheets of the brane. We suggest that this variation comes from nonminimal (gauge invariant) interaction of the adjoint scalar field with gauge fields. As a result of this interaction, the parameter of noncommutativity is actually determined by the VEV of the scalar field (for a similar construction of supersymmetric brane-antibrane configurations see Refs. $[8,9,10])$. Then, after the scalar potential of the double well shape is added, the resulting theory has a set of vacua with $U(\mathcal{N}) \times U(\mathcal{M})$ gauge groups. These vacua correspond to stacks of $\mathcal{N}$ branes and $\mathcal{M}$ antibanes. Also, there are BPS domain walls interpolating between $U(\mathcal{N}) \times U(\mathcal{M})$ and $U(\mathcal{N}+\mathcal{K}) \times U(\mathcal{M}+\mathcal{K})$ vacua for any $\mathcal{N}, \mathcal{M}$ and $\mathcal{K}$.

The rest of the paper is organized as follows. In section 2 we describe our setup and study the basic properties of the $U(\mathcal{N}) \times U(\mathcal{M})$ vacua. In section 3 we derive the BPS bound for the energy of the domain wall and solve the corresponding BPS equations. In section 4 we introduce the probe adjoint scalar field and study its spectrum in the domain wall background. This analysis supports our interperetation of the domain wall as a brane configuration shown in Fig. 1c. In the concluding section 5 we summarize our results, discuss the relation between our model and Matrix theory in curved background and speculate on some implications of our results for the brane world/mirror world scenario.

\section{The model}

Let us follow the steps oulined in the Introduction and construct the action of gauge theory on NC plane admitting domain walls between vacua with different gauge groups. To start with, let us consider the action of the pure NC $U(1)$ theory,

$$
S_{Y M}=-\frac{1}{4 g^{2}} \int d^{3} x F_{\lambda \nu}^{2}
$$


where $\lambda, \nu=t, x, y$. This action has the same form as that of the ordinary Yang-Mills theory. The only difference is that the Moyal $*$-product with the space-space noncommutativity,

$$
[x, y]_{*}=i \theta
$$

replaces the ordinary product of fields everywhere. Now, let us introduce a real scalar field $\phi$ in the adjoint representation of the gauge group with the double well potential

$$
S_{\phi}=\frac{1}{g^{2}} \int d^{3} x\left(\frac{1}{2}\left(D_{\nu} \phi\right)^{2}-\frac{\mu^{2}}{2}\left(\phi^{2}-v^{2}\right)^{2}\right)
$$

where again the $*$-product is assumed everywhere.

The next step is to add extra terms which will ensure that the noncommutativity parameter effectively changes its sign in the vacuum with $\phi=-v$. To this end, let us perform the Weyl map and write the gauge field action (1) in the operator language (see, e.g. Refs. [12] for details),

$$
S_{Y M}=\int d t \frac{2 \pi \theta}{g^{2}} \operatorname{Tr}\left(\frac{1}{2 \theta^{2}}\left(D_{0} X\right)^{2}+\frac{1}{2 \theta^{2}}\left(D_{0} Y\right)^{2}-\frac{1}{2 \theta^{4}}(i[X, Y]+\theta)^{2}\right),
$$

where covariant coordinates $X, Y$ are related to the gauge potentials as follows,

$$
\begin{aligned}
& X=\hat{x}+\theta \hat{A}_{y} \\
& Y=\hat{y}-\theta \hat{A}_{x} .
\end{aligned}
$$

Hats mean that we are working with operators in a Hilbert space. The $(x, y)$-component of the field strength tensor is equal to

$$
F_{x y}=-\frac{i}{\theta^{2}}[X, Y]-\frac{1}{\theta}
$$

In the operator formulation the only place where the sign of $\theta$ comes in is the last term in Eq. (3). To change this sign in the vacuum with $\phi=-v$ we introduce the extra $\phi$-dependence into this term, by multiplying $\theta$ by a factor $\phi / v$. Then the full action of the model takes the following form in the operator language

$$
\begin{aligned}
S=\int d t \frac{2 \pi \theta}{g^{2}} \operatorname{Tr} & \left\{\frac{1}{2 \theta^{2}}\left(D_{0} X\right)^{2}+\frac{1}{2 \theta^{2}}\left(D_{0} Y\right)^{2}+\frac{1}{2}\left(D_{0} \phi\right)^{2}\right. \\
& \left.-\frac{1}{2 \theta^{4}}\left(i[X, Y]+\frac{\theta}{v} \phi\right)^{2}-\frac{1}{2 \theta^{2}}(i[X, \phi])^{2}-\frac{1}{2 \theta^{2}}(i[Y, \phi])^{2}-\frac{\mu^{2}}{2}\left(\phi^{2}-v^{2}\right)^{2}\right\}
\end{aligned}
$$


As we discuss in more detail in section 5, in the spirit of Matrix model this action is naturally interpreted as describing dynamics of D0-branes in (curved) (3+1)-dimensional space-time. In this interpretation, operators $X, Y$ and

$$
Z \equiv \theta \phi
$$

represent spatial coordinates of D0-branes.

In what follows we work in the gauge $A_{0}=0$. Let us first classify time-independent vacuum solutions of the model. It follows immediately from Eq. (7) that the energy is minimum (zero), provided that

$$
\begin{gathered}
\phi^{2}=v^{2} \\
{[X, \phi]=[Y, \phi]=0} \\
{[X, Y]=i \frac{\theta}{v} \phi .}
\end{gathered}
$$

Solutions to Eqs. (8) are direct sums of any number of two basic representations defined on the Hilbert space of functions of one variable $q$,

$$
\begin{gathered}
\phi=v, \quad X=\hat{q}, \quad Y=-i \theta \frac{\partial}{\partial q} \\
\phi=-v, \quad X=-\hat{q}, \quad Y=-i \theta \frac{\partial}{\partial q} .
\end{gathered}
$$

From the Matrix model viewpoint, a direct sum of $\mathcal{N}$ representations of type (9a) and $\mathcal{M}$ representations of type $(9 \mathrm{~b})(\mathcal{N}-\mathcal{M}$ vacuum) corresponds to a system of $\mathcal{N}$ D2-branes and $\mathcal{M} \overline{\mathrm{D}} 2$-branes. The separation between D-branes and $\overline{\mathrm{D}}$-branes is given by

$$
a=\theta v
$$

The fact that under certain circumstances the brane-antibrane systems may exhibit no instability was observed in a similar context in Refs. [8, 9, 10].

It is worth noting that there exist various space-time formulations of the action (7). In particular, the original field-operator map used to rewrite $U(1)$ gauge theory (1) in the operator form (3), translates the action (7) into the following field theory action,

$$
S=\int d t d x d y \frac{1}{g^{2}}\left(-\frac{1}{4} F_{\lambda \nu}^{2}+\frac{1}{2}\left(D_{\nu} \phi\right)^{2}+\frac{1}{a} F_{x y}(\phi-v)-\frac{1}{2 a^{2}}(\phi-v)^{2}-\frac{\mu^{2}}{2}\left(\phi^{2}-v^{2}\right)^{2}\right),
$$

where, again, *-product is assumed everywhere. This space-time formulation is well suited for the study of the single D-brane vacuum (9a), which corresponds to $\mathcal{N}=1, \mathcal{M}=0$. 
Indeed, at the level of quadratic action the *-product is equivalent to the ordinary product, and the action (11) describes just a system of one vector and one scalar field with a certain (Lorentz-violating) mixing between them. Gauge field is zero in the vacuum (9a) and the VEV of the scalar field is equal to $v$. There are two propagating degrees of freedom in this vacuum with the following dispersion relations,

$$
\omega_{1,2}^{2}=k^{2}+\frac{m_{\phi}^{2}}{2} \pm \sqrt{\frac{m_{\phi}^{4}}{4}+\frac{k^{2}}{a^{2}}}
$$

where $\omega$ is energy, $k$ is the absolute value of the momentum, and

$$
m_{\phi}^{2}=\left(\frac{1}{\theta^{2} v^{2}}+4 \mu^{2} v^{2}\right) .
$$

At low momenta, $k \ll a m_{\phi}^{2}$, Eq. (12) reduces to

$$
\begin{aligned}
& \omega_{1}^{2}=m_{\phi}^{2}+k^{2}\left(1+\frac{1}{m_{\phi}^{2} a^{2}}\right) \\
& \omega_{2}^{2}=k^{2}\left(1-\frac{1}{m_{\phi}^{2} a^{2}}\right) .
\end{aligned}
$$

We see that Lorentz-violating effects are small at low energies provided that

$$
\mu \theta v^{2} \gg 1
$$

and the dispersion relations (14) describe one massless particle (photon) and one massive particle (scalar boson) in this limit. Note that the condition (15) is compatible with weak coupling regime in the naive commutative limit $\theta \rightarrow 0$ at small enough gauge coupling constant $g$ (we do not consider possible UV-IR mixing effects here). In what follows it is the limit

$$
\theta \rightarrow 0, \mu \theta v^{2} \rightarrow \infty
$$

which we refer to as the commutative limit.

From Eq. (6) we see that in the space-time formulation $(11), \overline{\mathrm{D}}$-brane vacuum $(\mathcal{N}=0$, $\mathcal{M}=1$ ) corresponds to the vacuum state with $\phi=-v$ and with non-zero magnetic field $F_{x y}=-2 / \theta$. On the other hand, in the operator language it is clear that physics in this vacuum is identical to physics in the D-brane vacuum. To see this explicitly one may replace the ordinary Weyl ordering by a different field-operator correspondence, defined as

$$
\hat{f} \leftrightarrow f(x, y) \equiv f_{W}(-x, y)
$$


where $f_{W}(x, y)$ is the Weyl symbol of operator $\hat{f}$. Also one changes relations (4), (5) between operators $X, Y$ and gauge fields, so that

$$
\begin{aligned}
& X=-\hat{x}-\theta \hat{A}_{y} \\
& Y=\hat{y}+\theta \hat{A}_{x} .
\end{aligned}
$$

Then it is straightforward to check, that in the space-time language the action (7) takes the same form (11) but with the different sign of the parameter $v$ and with the different sign of $\theta$ in the Moyal product. In this formulation the gauge field is zero in the $\bar{D}$-brane vacuum and non-zero in the D-brane vacuum. This argument supports our claim that the noncommutativity parameter changes its sign in the vacuum with $\phi=-v$.

Vacuum described by the direct sum of $\mathcal{N}$ D-brane representations (9a) appears as a highly inhomogeneous state in terms of the $U(1)$ theory (11). However, using the isomorphism between direct sum of $\mathcal{N}$ copies of the Fock space and a single Fock space one may map this vacuum into the vacuum with zero field in the $U(\mathcal{N})$ NC gauge theory, whose action is just a straightforward generalization of the action (11) to the case of $U(\mathcal{N})$ gauge group (cf. Refs. [1]-[4], [6]).

Similar reinterpretation of vacua where both D- and $\bar{D}$-branes are present is somewhat more subtle. Indeed, we saw that for D-brane sector, it is natural to use the space-time formulation with positive $\theta$, while for $\overline{\mathrm{D}}$-brane sector, the formulation with negative value of $\theta$ is more appropriate. However, when both sectors are present, there appear bifundamental fields charged under gauge groups of both sectors. To understand what happens in this case, it is instructive to study the physics of the D-D pair $(\mathcal{N}=1, \mathcal{M}=1)$.

Let us use, for space-time interpretation, the formulation with positive $\theta$. The action (7) then takes the form of the action of $\mathrm{NC} U(2)$ gauge theory, which is similar to (11) with the only difference that all fields are now $2 \times 2$ matrices and trace over $U(2)$ indices is assumed. Background corresponding to D-D configuration has the following form (cf. Eqs. (9)),

$$
\phi=\left(\begin{array}{cc}
v & 0 \\
0 & -v
\end{array}\right), \quad X=\left(\begin{array}{cc}
q & 0 \\
0 & -q
\end{array}\right), \quad Y=\left(\begin{array}{cc}
-i \theta \frac{\partial}{\partial q} & 0 \\
0 & -i \theta \frac{\partial}{\partial q}
\end{array}\right) \text {. }
$$

It breaks $U(2)$ gauge group down to $U(1) \times U(1)$. Magnetic field in this background is non-zero:

$$
F_{x y} \equiv-\frac{1}{\theta^{2}}(i[X, Y]+\theta)=\left(\begin{array}{cc}
0 & 0 \\
0 & -\frac{2}{\theta}
\end{array}\right) .
$$

The block-diagonal structure of background (17) ensures that spectra of diagonal components of gauge fields and field $\phi$ are not modified and are given by Eqs. (14). On the other hand, 
off-diagonal components feel both the VEV of the field $\phi$ and the magnetic field (18). So, one expects them to have Landau spectrum of energies with cyclotron frequency of order $\frac{1}{\theta}$ and a constant piece of order $v^{2}$ (cf. Eq. (25) below). Instead of performing explicit analysis of the gauge sector, we confirm these statements by working out the spectrum of a probe adjoint field $\chi$ in the D-D background. This spectrum shares essential features of the gauge field spectrum, while its evaluation is more transparent.

The field equation for the field $\chi$ reads

$$
-D_{0}^{2} \chi-\frac{1}{\theta^{2}}[X,[X, \chi]]-\frac{1}{\theta^{2}}[Y,[Y, \chi]]-[\phi,[\phi, \chi]]=0 .
$$

Note that besides the interaction with gauge fields we introduce interaction of the probe field with the scalar field $\phi$. The latter interaction has the form typical for the adjoint fields in the gauge theories residing on the stacks of D-branes, and enables one to interpret the field $\chi$ as the (matrix valued) coordinate of the brane system in the extra transverse direction. The diagonal structure of the background fields (17) implies that there is no mixing between different components in $2 \times 2$ matrix representation of $\chi$,

$$
\chi=\left(\begin{array}{cc}
\alpha & \gamma \\
\gamma^{+} & \beta
\end{array}\right) \text {. }
$$

Equations for diagonal components $\alpha$ and $\beta$ following from (19), (17) coincide with the equations for free massless scalar field in the operator language. They have plane wave solutions

$$
\alpha, \beta \propto \mathrm{e}^{i\left(\omega t+k_{x} \hat{x}+k_{y} \hat{y}\right)}
$$

with the massless dispersion relations

$$
w^{2}=k_{x}^{2}+k_{y}^{2}
$$

In the string interpretation these components correspond to the strings with both ends on one of the branes.

Let us now consider off-diagonal sector $\gamma$ which corresponds to strings stretched between the brane and the antibrane. It is convenient to consider $\gamma$ as an operator in the space of functions of one variable $q$ (cf. Eqs. (9)) and rewrite Eq. (19) in terms of the kernel $\gamma\left(q, q^{\prime}\right)$,

$$
-\partial_{0}^{2} \gamma-\frac{\left(q+q^{\prime}\right)^{2}}{\theta^{2}} \gamma+\left(\frac{\partial}{\partial q}+\frac{\partial}{\partial q^{\prime}}\right)^{2} \gamma-4 v^{2} \gamma=0 .
$$

Let us search for solutions of Eq. (22) of the following form,

$$
\gamma\left(q, q^{\prime}\right)=\mathrm{e}^{i \omega t} \bar{\gamma}(\bar{q}) \delta\left(u-\theta k_{y}\right)
$$


where $\bar{q}=\left(q^{\prime}+q\right) / 2$ and $u=q^{\prime}-q$. The $\delta$-function in Eq. (23) is the $q$-representation of the operator $\mathrm{e}^{i k_{y} \hat{y}}$, so Ansatz (23) corresponds to the Fourier decomposition in the $y$-direction. Then one arrives at the following equation for the function $\bar{\gamma}(\bar{q})$

$$
\omega^{2} \bar{\gamma}=-\frac{\partial^{2} \bar{\gamma}}{\partial \bar{q}^{2}}+\left(4 v^{2}+\frac{4 \bar{q}^{2}}{\theta^{2}}\right) \bar{\gamma} .
$$

Eq. (24) coincides with the eigenfunction equation for a quantum mechanical oscillator. It yields the Landau spectrum,

$$
\omega^{2}=4 v^{2}+\frac{4}{\theta}\left(n+\frac{1}{2}\right), \quad n=0,1,2, \ldots
$$

Note that for off-diagonal modes $\gamma$, the variable $u / 2$ plays the role of the $x$-coordinate of the string center, while $2 \bar{q}$ is the difference between coordinates of the ends of the string; this is due to our choice of the background configuration (17). Consequently, the proper interpretation of $k_{y}$ is that it determines the $x$-coordinate of the excitation, namely

$$
x_{0}=\frac{k_{y} \theta}{2} .
$$

The constant piece $4 v^{2}$ in the expression for energy (25) is generated by the VEV of the field $\phi$. In the string language its presence is explained by the fact that the minimal length of the string stretched between brane and antibrane in the D-D system is proportional to $v$.

Similar analysis applies to systems with larger number of branes. The vacuum with $\mathcal{N}$ branes and $\mathcal{M}$ antibranes can be thought of as the trivial vacuum in the $U(\mathcal{N}) \times U(\mathcal{M}) \mathrm{NC}$ gauge theory with opposite signs of noncommutativity parameter in different sectors and with extra bifundamental fields interacting with both sectors. The latter fields exhibit the spectrum of Landau type; they become heavy and decouple in the commutative limit.

\section{Domain wall}

We turn now to the construction of a domain wall separating vacua with different gauge groups. For concreteness let us concentrate on the $U(1)-U(2) \times U(1)$ domain wall (on the one side of the wall the gauge group is $U(1)$, while one the other side it is $U(2) \times U(1)$ ). Energy functional for static field configurations following from the action (7), can be written as

$$
\begin{aligned}
E=\frac{2 \pi \theta}{g^{2}} \operatorname{Tr}\{ & \frac{1}{2 \theta^{2}}(i[X, \phi])^{2}+\frac{1}{2 \theta^{4}}\left(i[X, Y]+\frac{\theta}{v} \phi\right)^{2} \\
& \left.+\frac{1}{2}\left(\frac{i}{\theta}[Y, \phi] \pm \mu\left(\phi^{2}-v^{2}\right)\right)^{2} \mp \frac{i \mu}{2 \theta}\left[\phi^{2} Y+Y \phi^{2}-2 v^{2} Y, \phi\right]\right\}
\end{aligned}
$$


This implies the BPS bound for energy,

$$
E \geq \frac{\mu v^{2}}{g^{2}}|Q|
$$

where

$$
Q=i \pi \operatorname{Tr}\left[\frac{\phi^{2} Y+Y \phi^{2}}{v^{2}}-2 Y, \phi\right]
$$

is a topological charge (being a trace of a commutator, $Q$ does not change under local variations of the fields). Let us search for solutions saturating the bound (28) in the topological sector with a given charge $Q$. They obey a system of BPS equations following from Eq.(27),

$$
\begin{aligned}
& {[X, \phi]=0} \\
& i[X, Y]+\frac{\theta}{v} \phi=0 \\
& \frac{i}{\theta}[Y, \phi] \pm \mu\left(\phi^{2}-v^{2}\right)=0 .
\end{aligned}
$$

These equations can be considered as defining an algebra with three generators $X, Y, \phi$. Each solution of Eqs. (30) decomposes into a direct sum of operators acting in irreducible representations of this algebra. Thus, let us classify irreducible representations of the algebra (30).

Without loss of generality we work in the $x$-representation. Namely, we assume that operators $X, Y, \phi$ act in the Hilbert space of functions $\Psi(x)$ of a single variable $x$, and

$$
(X \Psi)(x)=x \Psi(x)
$$

From Eq. (30a) it follows that

$$
(\phi \Psi)(x)=\phi(x) \Psi(x)
$$

where $\phi(x)$ is some yet unknown function. Eq. (30b) yields

$$
(Y \Psi)(x)=-i \frac{\theta}{v} \phi(x) \frac{\partial \Psi(x)}{\partial x}
$$

Then Eq. (30c) (where, to be specific, we consider the upper sign) reads

$$
\frac{1}{v} \phi \frac{\partial \phi}{\partial x}+\mu\left(\phi^{2}-v^{2}\right)=0
$$

This equation has three different bounded solutions:

a) $\phi=v$; in this case $-\infty<x<+\infty, Y=-i \theta \frac{\partial}{\partial x}$, and, as it can be easily shown, the 


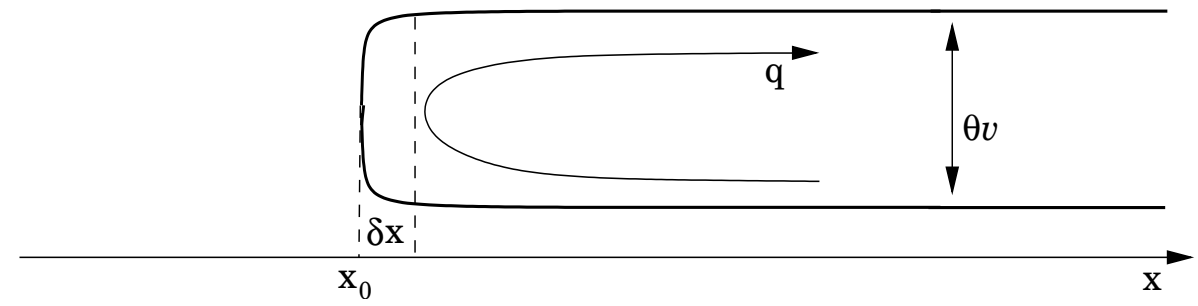

Figure 2: Geometrical meaning of the parameters of the folded brane.

topological charge $Q$ is zero. This solution corresponds to a single $D$-brane located at the point $Z=\theta v$ in the transverse direction.

b) $\phi=-v$; in this case $-\infty<x<+\infty$, and $Y=i \theta \frac{\partial}{\partial x}$. The topological charge $Q$ is zero as in the previous case. This solution corresponds to an antibrane located at $Z=-\theta v$.

c) $\phi= \pm v \sqrt{1-\mathrm{e}^{-2 \mu v\left(x-x_{0}\right)}}, x_{0} \leq x$. This solution describes a brane folded twice along the $x$-direction, with the constant $x_{0}$ corresponding to the position of the tip of the fold (see Fig. 2). It is straightforward to check that $Q \neq 0$ in this case. It is convenient to express this solution in the $q$-representation, where the variable $q$ is a coordinate along the fold; it is related to $x$ by

$$
d q=\frac{v d x}{\phi(x)}
$$

Straightforward calculation yields

$$
\begin{aligned}
\phi & =\phi(q) \equiv v \operatorname{th}(\mu v q) \\
X & =x(q) \equiv \frac{1}{\mu v} \ln (2 \operatorname{ch}(\mu v q))+x_{0} \\
Y & =-i \theta \frac{\partial}{\partial q} .
\end{aligned}
$$

The geometrical meaning of the coordinates $x$ and $q$ is illustarted in Fig. 2. The width of the tip of the fold can be read off from Eqs. (36a), (36b),

$$
\delta x \approx \frac{1}{\mu v} .
$$

Note that the condition (15) implies that the distance between two sheets of the folded brane in the transverse direction, which is equal to $\theta v$, is much larger than the width of the tip.

The $U(1)-U(2) \times U(1)$ domain wall is obtained by taking the direct sum of representations 
(a) and $(c)$,

$$
\phi=\left(\begin{array}{cc}
v & 0 \\
0 & \phi(q)
\end{array}\right), \quad X=\left(\begin{array}{cc}
q & 0 \\
0 & x(q)
\end{array}\right), \quad Y=\left(\begin{array}{cc}
-i \theta \frac{\partial}{\partial q} & 0 \\
0 & -i \theta \frac{\partial}{\partial q}
\end{array}\right),
$$

where functions $\phi(q)$ and $x(q)$ are given by Eqs. (36a), (36b). The solution (38) describes a wall extending along $y$-direction, which is located at the position $x_{0}$ on the $x$-axis. To calculate the tension of the domain wall let us plug the solution (38) into the expression for the topological charge (29) and use Eqs. (36a), (36c). Then, from the BPS bound (28) one obtains for the energy

$$
\begin{aligned}
E & =-\frac{2 \pi \theta \mu}{g^{2}} \operatorname{Tr}\left\{\frac{\partial \phi}{\partial q}\left(\phi^{2}-v^{2}\right)\right\} \\
& =-\frac{\mu}{g^{2}} \int d y d q \frac{\partial \phi}{\partial q}\left(\phi^{2}-v^{2}\right)=\int d y \frac{4 \mu v^{3}}{3 g^{2}},
\end{aligned}
$$

where in the second equality we used the relation $2 \pi \theta \operatorname{Tr}=\int d y d q$. Thus, the tension of the wall equals

$$
\sigma=\frac{4 \mu v^{3}}{3 g^{2}} .
$$

In the commutative limit (16) the width of the wall can be kept finite, while its tension tends to infinity.

Construction presented above is straightforward to generalize to the case of $U(\mathcal{N}) \times$ $U(\mathcal{M})-U(\mathcal{N}+\mathcal{K}) \times U(\mathcal{M}+\mathcal{K})$ domain walls: one takes direct sum of $\mathcal{N}$ representations of type $(a), \mathcal{M}$ representations of type $(b)$ and $\mathcal{K}$ representations of type $(c)$. Let us also point out that though in this section we have concentrated on domain walls extending along $y$-axis, this direction is by no means preferred from the point of view of the action (7). Performing rotation of the solution constructed in this section, one can obtain domain walls oriented arbitrarily in the $x y$-plane.

\section{Adjoint scalar in the domain wall background}

The purpose of this section is to check the intuition coming from D-brane picture and to confirm that the solution constructed in the previous section describes a domain wall between regions with different gauge theories. For concreteness, we concentrate on the case of the $U(1)-U(2) \times U(1)$ domain wall. Generalization of our analysis to $U(\mathcal{N}) \times U(\mathcal{M})-U(\mathcal{N}+$ $\mathcal{K}) \times U(\mathcal{M}+\mathcal{K})$ domain wall solutions is straightforward. 
Again, as in section 2, for the sake of simplicity let us consider massless adjoint scalar field $\chi$ as a probe and study its spectrum in the domain wall background. Throughout this section we assume that the condition (15) is satisfied. This is the case in which our results have the most transparent physical interpretation.

Field equation for the field $\chi$ is given by Eq. (19) with background fields (38). For definiteness we take $x_{0}=0$, i.e. consider a domain wall with the tip at $x=0$. As in section 2 , we take $\chi$ in the form (20). Diagonal structure of (38) implies that fields $\alpha, \beta, \gamma$ of this decomposition do not mix.

Analysis of the spectrum in the $\alpha$-sector literally repeats that of section 2 . Thus, the field $\alpha$ describes massless particles that are able to move freely from $x \rightarrow-\infty$ to $x \rightarrow+\infty$ and do not feel the presence of the domain wall at all. These modes describe excitations of strings with both ends on the straight D-brane.

On the other hand, fields of the $\beta$-sector do not interact with the straight brane, and live completely on the folded brane. From Eqs. (19), (38) we get the following equation for the kernel $\beta\left(q, q^{\prime}\right)$,

$$
-\partial_{0}^{2} \beta-\left(\frac{\left(x(q)-x\left(q^{\prime}\right)\right)^{2}}{\theta^{2}}+\left(\phi(q)-\phi\left(q^{\prime}\right)\right)^{2}\right) \beta+\left(\frac{\partial}{\partial q}+\frac{\partial}{\partial q^{\prime}}\right)^{2} \beta=0 .
$$

Using the same Ansatz as in section 2,

$$
\beta\left(q, q^{\prime}\right)=\mathrm{e}^{i \omega t} \bar{\beta}(\bar{q}) \delta\left(u-\theta k_{y}\right)
$$

where $\bar{q}=\left(q^{\prime}+q\right) / 2, u=q^{\prime}-q$, we arrive at the following Schrödinger-type equation for $\bar{\beta}$,

$$
\omega^{2} \bar{\beta}=-\frac{\partial^{2} \bar{\beta}}{\partial \bar{q}^{2}}+V_{\beta}\left(\bar{q}, k_{y}\right) \bar{\beta} .
$$

Here, the potential $V_{\beta}$ is given by

$$
V_{\beta}\left(\bar{q}, k_{y}\right)=\left(\frac{1}{\mu v \theta} \ln \frac{\operatorname{ch} \mu v\left(\bar{q}-\frac{\theta k_{y}}{2}\right)}{\operatorname{ch} \mu v\left(\bar{q}+\frac{\theta k_{y}}{2}\right)}\right)^{2}+v^{2}\left(\operatorname{th} \mu v\left(\bar{q}-\frac{\theta k_{y}}{2}\right)-\operatorname{th} \mu v\left(\bar{q}+\frac{\theta k_{y}}{2}\right)\right)^{2} .
$$

This potential is shown in Fig. 3 for three different regions of parameter $k_{y}$. In all three cases it approaches $k_{y}^{2}$ as $\bar{q} \rightarrow \pm \infty$. Thus, asymptotically, the solution to Eq. (43) is given by plane waves $\bar{\beta} \propto \mathrm{e}^{ \pm i k_{x} \bar{q}}$ with dispersion relation (21). Two regions, $(\bar{q}>0)$ and $(\bar{q}<0)$ correspond to the upper and lower sheets of the folded brane, respectively. We will refer to modes of the field $\beta$ living on the upper (lower) sheet as $\beta_{+}\left(\beta_{-}\right)$sector. This terminology 


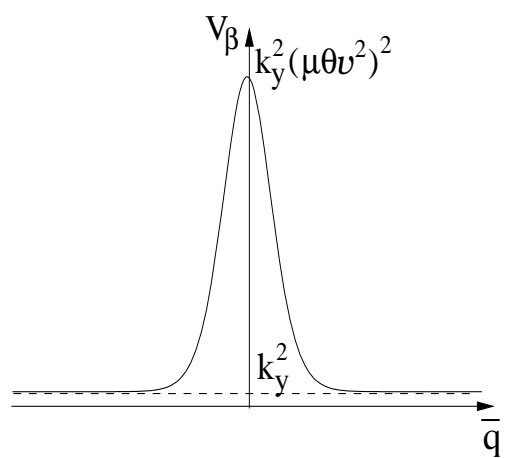

a

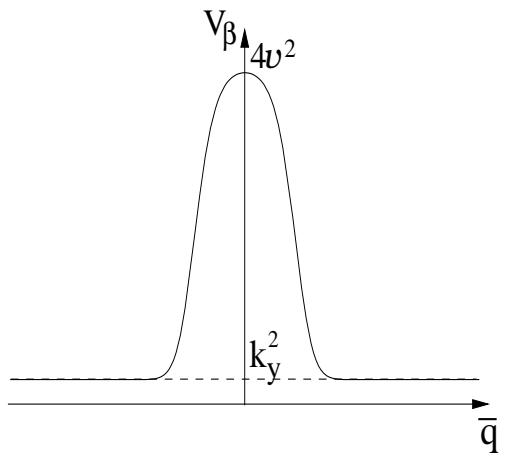

$\mathrm{b}$

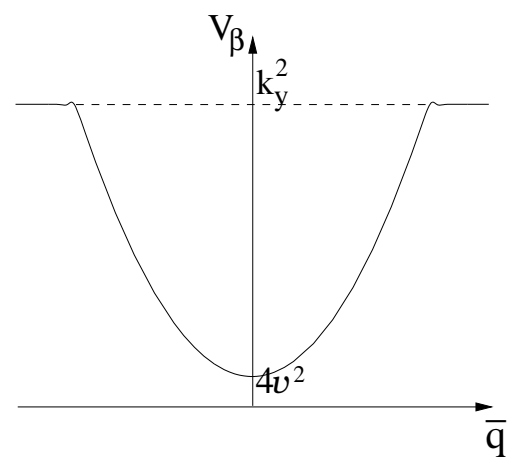

C

Figure 3: Potential $V_{\beta}$ as a function of $\bar{q}$ for three regions of parameter $k_{y}$ :

a) $k_{y} \ll \frac{1}{\mu \theta v}$, b) $\frac{1}{\mu \theta v} \ll k_{y} \ll v$, c) $v \ll k_{y}$.

does not imply that $\beta_{+}$and $\beta_{-}$sectors are disconnected: as we will see immediately there are modes that can propagate round the tip of the folded brane from one sheet to another.

The behavior of the potential $V_{\beta}$ in the region near the tip, $\bar{q} \approx 0$, depends on the value of $k_{y}$. For $k_{y} \ll \frac{1}{\mu \theta v}$ (see Fig. 3a) it has a sharp peak around $\bar{q}=0$ with the height $k_{y}^{2}\left(\mu \theta v^{2}\right)^{2} \gg k_{y}^{2}$. This means that modes with small momentum along $x$-axis are reflected backwards from the tip, their propagation through the tip being suppressed. However, there are modes that can propagate round the tip without suppression, namely, those whose wavevector is almost perpendicular to the tip, $k_{x}^{2}>k_{y}^{2}\left(\mu \theta v^{2}\right)^{2}$.

When $\frac{1}{\mu \theta v} \ll k_{y} \ll v$, the potential barrier separating $\beta_{+}$and $\beta_{-}$sectors is still present, but now its height is equal to $4 v^{2}$ (see Fig. $3 \mathrm{~b}$ ).

Behavior of $V_{\beta}(\bar{q})$ is qualitatively different for $v \ll k_{y}$ (Fig. 3c). The potential has a dip at the origin with minimal value $4 v^{2}$. Around this minimum it can be approximated as follows,

$$
V_{\beta} \approx 4 v^{2}+\frac{4 \bar{q}^{2}}{\theta^{2}}
$$

Thus the energy spectrum for low-lying modes is given by Eq. (25) in this case. We will refer to these modes as $\beta_{*}$-sector.

Many of these features have physical explanation in the dipole interpretation $[13,14]$. Namely, one may consider excitations in the NC theory as dipoles (strings) whose length in, say, $q$-direction is proportional to the momentum in the perpendicular $y$-direction,

$$
\Delta q=\theta k_{y}
$$




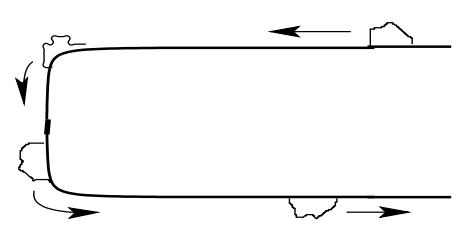

a

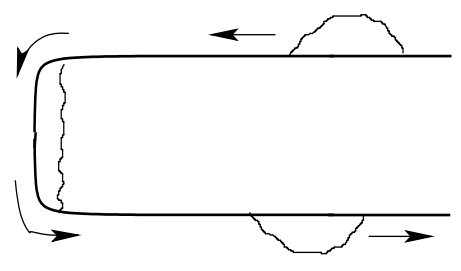

$\mathrm{b}$

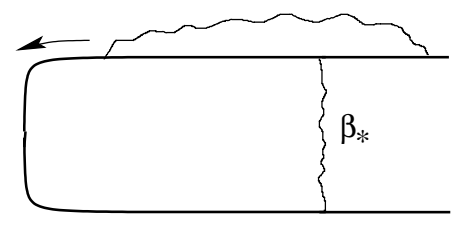

$\mathrm{C}$

Figure 4: Stringy interpretation of small perturbations in the folded brane background.
a) $k_{y} \ll \frac{1}{\mu \theta v}$,
b) $\frac{1}{\mu \theta v} \ll k_{y} \ll v$,
c) $v \ll k_{y}$.

Then in the first kinematical region, $k_{y} \ll 1 /(\mu \theta v)$, the length of the string in $q$ direction is much smaller than the characteristic curvature radius of the tip. Both ends of such string enters into the curved region simultaneously, see Fig. 4a. The fact that the height of the potential barrier grows with $k_{y}$ is easy to understand - the longer the string is, the more difficult it is for this string to turn round the tip. It would be interesting to calculate the coefficient of proportionality between $k_{y}$ and the height of the barrier in the string language.

In the second region, the situation changes. Here the length of the string is larger than the curvature radius of the tip. The ends of such string do not turn round the tip at one time anymore. Instead, first the left end turns round the tip, and only after it reaches the second sheet, the right end follows, see Fig. 4b. In this regime the height of the barrier does not depend on $k_{y}$. Rather, it is given by the energy of the string stretched between the two sheets, which is equal to $2 v$.

Finally, the third region corresponds to strings with separation between their ends in the $q$-direction much larger then the distance between the two sheets of the fold. Here, two types of strings exist. First, there are very long strings that do not experience any potential barrier while propagating from one sheet to another. At the same time new type of states appear $\left(\beta_{*}\right.$-sector). These are strings stretched between different sheets of the folded brane, see Fig. 4c. They correspond to the heavy states (25) present in the spectrum of D-D system. Note that for these strings, $k_{y}$ measures the distance from the tip of the fold. Here the difference between $q$ and $x$ coordinates is crucial. Namely, for these strings the difference between $q$ coordinates of their ends is still given by Eq. (46), so these strings exist only far enough from the tip. However, as $q$ and $-q$ correspond to the same value of the physical $x$ coordinate, the actual length of these strings can be as small as the distance $2 \theta v$ between the two sheets of the folded brane. 
To summarize, far from the tip, we see that the spectrum of the folded brane reproduces the spectrum of the D-D system.

Let us now consider the $\gamma$-component of the field $\chi$ (see Eq. (20)). This component corresponds to strings with one end on the straight brane and the other on the folded one. Here we have the following equation for the kernel $\gamma\left(q, q^{\prime}\right)$,

$$
-\partial_{0}^{2} \gamma-\left(\frac{\left(q-x\left(q^{\prime}\right)\right)^{2}}{\theta^{2}}+\left(v-\phi\left(q^{\prime}\right)\right)^{2}\right) \gamma+\left(\frac{\partial}{\partial q}+\frac{\partial}{\partial q^{\prime}}\right)^{2} \gamma=0 .
$$

Using Ansatz analogous to (42) we get

$$
\omega^{2} \bar{\gamma}=-\frac{\partial^{2} \bar{\gamma}}{\partial \bar{q}^{2}}+V_{\gamma}\left(\bar{q}, k_{y}\right) \bar{\gamma}
$$

where

$$
\begin{aligned}
V_{\gamma}\left(\bar{q}, k_{y}\right)=\frac{1}{\theta^{2}}\left\{\bar{q}-\frac{\theta k_{y}}{2}-\frac{1}{\mu v} \ln (2 \operatorname{ch} \mu v\right. & \left.\left.\left(\bar{q}+\frac{\theta k_{y}}{2}\right)\right)\right\}^{2} \\
& +v^{2}\left(1-\operatorname{th} \mu v\left(\bar{q}+\frac{\theta k_{y}}{2}\right)\right)^{2}
\end{aligned}
$$

Qualitative behavior of the potential $V_{\gamma}$ in two different regions of the parameter $k_{y}$ is shown in Fig. 5. In both cases its asymptotics are

$$
\begin{array}{cc}
V_{\gamma} \rightarrow k_{y}^{2}, & \bar{q} \rightarrow+\infty \\
V_{\gamma} \sim \frac{4 \bar{q}^{2}}{\theta^{2}}, & \bar{q} \rightarrow-\infty
\end{array}
$$

Thus, $\gamma$-sector contains a sector of plane waves living in the region $\bar{q}>0$. Let us call these modes $\gamma_{+}$-sector. They correspond to strings with one end on the straight brane and the other on the upper sheet of the folded brane. One of the ends of these strings always has positive $x$-coordinate. This explains why the $\gamma_{+}$-modes cannot penetrate into the region of negative $\bar{q}$, which corresponds to negative $x$-coordinate on the straight brane.

When $k_{y}<0, \quad\left|k_{y}\right| \gg v$, the potential $V_{\gamma}$ has a dip near the origin (Fig. 5b) similar to the case of $\beta$-sector (cf. Fig. 3c). In the vicinity of the minimum one has

$$
V_{\gamma} \approx 4 v^{2}+\frac{4 \bar{q}^{2}}{\theta^{2}}
$$

The energy spectrum of low-lying modes in this case is again given by Eq. (25). These modes correspond to strings stretched between the straight brane and the lower sheet of the folded one. We denote this sector by $\gamma_{*}$. 


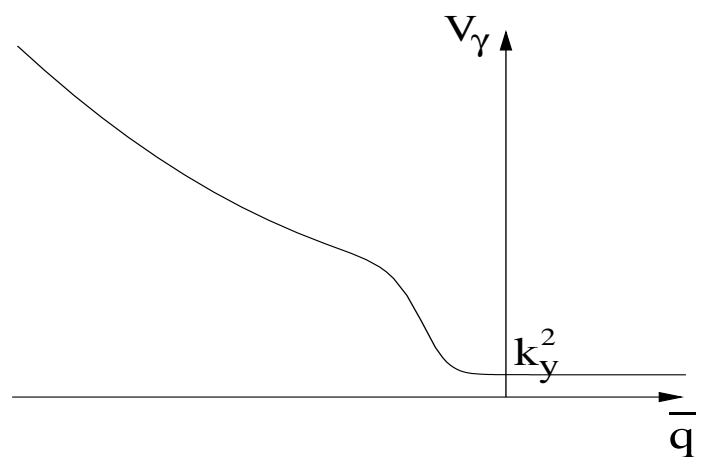

a

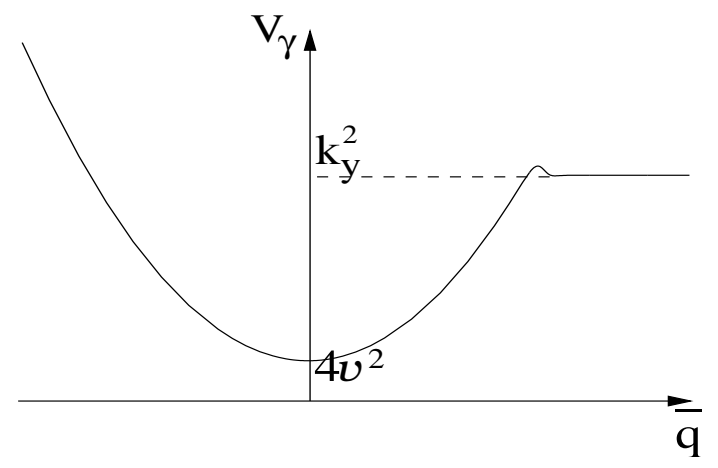

$\mathrm{b}$

Figure 5: Potential $V_{\gamma}$ as a function of $\bar{q}$ for two regions of parameter $k_{y}$ :

a) $k_{y}>-v$, b) $k_{y}<0, \quad\left|k_{y}\right| \gg v$.

Let us summarize the results of this section and give an interpretation to the spectrum of the adjoint field $\chi$ from the viewpoint of $(2+1)$ dimensional field theory. In the region $x \rightarrow-\infty$ there is one massless field $\alpha$. This means that in this region we have $U(1)$ gauge theory. In the region $x \rightarrow+\infty$ this field, together with $\beta_{+^{-}}, \gamma_{+}$-fields, form the adjoint multiplet of the $U(2)$ gauge group

$$
\left(\begin{array}{cc}
\alpha & \gamma_{+} \\
\gamma_{+}^{+} & \beta_{+}
\end{array}\right)
$$

Note that the field $\alpha$ propagates freely from $x=-\infty$ to $x=+\infty$. Thus, the $U(1)$ and $U(2)$ gauge theories on either side of the wall are connected even at low energies. In addition there is another massless field $\beta_{-}$at $x \rightarrow+\infty$. This is a signal that an additional $U(1)$ gauge group is present in this region. Let us stress that this $U(1)$ gauge group is different from the $U(1)$ in the region $x \rightarrow-\infty$. Finally, there are high-energy modes $\beta_{*}, \gamma_{*}$ with the Landau spectrum (25) living at large enough positive $x \gtrsim \theta v$.

These results confirm our statement, that the solution obtained in the previous section describes a domain wall between regions with $U(1)$ and $U(2) \times U(1)$ gauge theories.

\section{Conclusions and discussion}

From the field theory viewpoint we presented an example of a gauge theory with adjoint scalar on NC plane, such that the rank and the type of the gauge group in it are not fixed, but can change dynamically. In particular, they can vary along the plane. We constructed 
explicit solutions in the form of domain walls interpolating between regions in space with different gauge groups. Though our model is formulated in $(2+1)$ dimensions, it can be easily generalized to higher dimensional space-time by adding an arbitrary number of commuting space-like dimensions. Then, the higher dimensional model possesses domain wall solutions independent of the commuting coordinates.

The fact that the gauge groups on different sides of the wall are different was confirmed by explicit evaluation of the spectrum of a probe adjoint field. Namely, we demostrated that its excitations with equal momentum and energy form adjoint multiplets of different gauge theories on the two sides of the wall. As in the case of domain walls on the fuzzy cylinder [6] a similar analysis can be performed for fundamental probe fields and for the gauge fields themselves. An outcome of this analysis is that there are always modes charged under the gauge group, that can freely propagate through the wall. This holds even for the lowenergy part of the spectrum whose dynamics on either side of the wall is desribed (barring potential UV/IR mixing effects) by commutative gauge theory. These results indicate that (non)commutative $U(\mathcal{N})$ gauge theories may be thought of as dynamically connected sectors of a single theory. It is interesting to explore this relationship at the quantum level. We leave this investigation for future studies.

We have seen above that many properties of the model considered in this paper have natural physical interpretation in stringy terms. This suggests that there should be a way to embed domain walls discussed in this paper into the context of string/M-theory. Let us briefly present arguments indicating that such embedding is quite plausible. These arguments are parallel to those presented in the case of the domain wall on the fuzzy cylinder [6]. We refer an interested reader to Ref. [6] for more details.

Let us recall that according to the BFSS conjecture [11], the dynamics of the M-theory in the infinite momentum frame is described by the large- $N$ limit of the BFSS Matrix model. The latter is supersymmetric quantum mechanics described by the following Lagrangian (see, e.g., Ref. [15] for a review)

$$
\left.L=\frac{1}{2 R} \operatorname{Tr}\left\{\dot{X}^{i} \dot{X}^{i}+\frac{1}{2}\left[X^{i}, X^{j}\right]^{2}+\text { (fermions }\right)\right\},
$$

where $X^{i}(i=1, \ldots, 9)$ are real-valued $N \times N$ matrices subject to the constraint

$$
\left[\dot{X}^{i}, X^{i}\right]=0
$$

The matrices $X^{i}$ are (matrix-valued) coordinates of D0 branes (in string units $l_{s}=1$ ) which are conjectured to be the partons of M-theory. The parameter $R$ is the string coupling 
constant which is interpreted as the compactification radius of 11-dimensional M-theory to ten dimensions.

Here we would like to suggest that domain walls studied in this paper may be obtained as solutions of the Matrix model in curved backgrounds. Our proposal is based on the observation that if one sets $X^{i}=0$ for $i \geq 4$ in the Matrix model Lagrangian, one arrives at the action very similar to the action (7) where $X, Y$ and $Z=\theta \phi$ correspond to matrix coordinates $X_{1}, X_{2}, X_{3}$, string length $l_{s}$ is equal to the scale of noncommutativity, $l_{s}=\theta^{1 / 2}$ and $R=g^{2} \theta$. The only difference is that in our theory we have a potential for the field $\phi$ and that extra $\phi$-dependence is present in the first term $\frac{\theta}{v} \phi$ in the second line of Eq. (7). Terms of this structure are expected to appear in the Lagrangians of Matrix models corresponding to a large class of curved backgrounds of M-theory [15].

It is worth noting, that generalization of the Matrix model Lagrangian (54) to arbitrary curved background is not known (see Ref. [16] for a discussion of this problem). However, there is a proposal [17] on how to modify the Lagrangian of the Matrix model to incorporate the effect of arbitrary weakly curved background independent of the light cone coordinate $x^{-}$. This proposal makes it natural to consider [6] the metric of the eleven-dimensional pp-wave

$$
d s^{2}=-2 d x^{+} d x^{-}+\sum d x^{l} d x^{l}-H\left(x^{l}\right)\left(d x^{+}\right)^{2} .
$$

In order to be a legitimate background of the M-theory, at least in the supergravity approximation, this metric should be supplemented by an appropriate three-form field to satisfy the equations of eleven-dimensional supergravity. This class of solutions was found in Ref. [18] and homogeneous pp-wave considered recently in Ref. [19] is a particular example of the background from this class.

Then, following the prescription of Ref. [17], it is straightforward to find various backgrounds in which the action of the BFSS Matrix model contains the terms present in Eq. (7). For instance, the metric of the form (55) with the function

$$
H=\frac{1}{a^{2}} z^{2}+\mu^{2}\left(z^{2}-a^{2}\right)^{2}+2 \mu^{2} a^{2} x_{4}^{2}+\mu^{2} x_{4}^{4}
$$

supplemented with the following three-form

$$
A=\frac{1}{a}\left(z d x_{+} \wedge d x \wedge d y+x_{4} d x_{+} \wedge d x \wedge d y\right)+\sqrt{12} \mu z x_{4} d x_{+} \wedge d x_{5} \wedge d x_{6}
$$

will do the job ${ }^{1}$. It is worth noting that with this choice of background some extra terms besides those of Eq. (7) will appear in the Lagrangian of the Matrix model. However, these

\footnotetext{
${ }^{1}$ Note, that unlike in the previous section, we are working in the string units $\theta=l_{s}^{2}=1$.
} 
terms are at least quadratic in the coordinates $X_{i}$ with $i>3$ and positive definite at the quadratic level. Consequently, they affect neither existence nor perturbative stability of the brane configurations considered above. Thus, it is natural to expect that configurations of this type naturally appear in a large class of curved backgrounds.

Clearly, our arguments rely on the approximation of weakly curved background; one may hope that they apply beyond this approximation, especially taking into account that pp-wave backgrounds similar to those we discussed here were shown to be exact string backgrounds $[20,21]$. We leave aside an issue of the supersymmetrization of the domain walls, though the BPS property suggests that it should be possible.

Finally, note that the folded brane involved into construction of the domain wall can be considered by itself and provides an explicit realization of the old idea of "mirror world" [22] (for recent review see Ref. [23]). Similar idea was put forward in Ref. [24] in the context of brane world models under the name of "many fold Universe". According to this scenario our world is a brane folded several times inside the bulk space. Matter residing on the different sheets of the folded brane is microphysically identical to the ordinary matter (in our case these two types of matter are represented by $\beta_{+}$and $\beta_{-}$sectors), but at low energies only gravitational interaction is possible between them. As a result, matter on a sheet different from ours may be observed as dark matter.

Some possible phenomenological aspects of this scenario were considered in Ref. [24]. Here we would like to mention just one more. As we saw in section 4, there are high energy modes $\left(\gamma_{*}\right.$ and $\left.\beta_{*}\right)$ corresponding to strings stretched between different sheets of the folded brane. At high enough energy, production of these modes and, as a result, direct connection between different sheets is possible. ${ }^{2}$

With a bit of fantasy one may envisage an exciting possibility that an intelligent life exists in the Solar system, but on a different sheet of our brane. Then, creating strings stretched between the two sheets of the brane at future accelerators, such as $\mathrm{LHC}^{3}$, one can establish direct communication with this civilization, with information transfer in the form, say, of the Morse code. Equally exciting is to look for messages from this civilization in the ultra-high energy cosmic ray data. Clearly, this possibility appears extremely unlikely. The only argument we may present in favor of this idea is due to Freeman Dyson [25]: "astronomy

\footnotetext{
${ }^{2}$ Note that in our model the dispersion relation for these modes is highly Lorentz-noninvariant. It is interesting to understand whether this is a generic phenomenon and what are the phenomenological implications of this violation of Lorentz invariance.

${ }^{3} \mathrm{LHC}=$ Large Humanoid Communicator.
} 
teaches us that we should look not for those things which are likely, but for those which are detectable".

\section{Acknowlegements}

We are indebted to D. Gorbunov and V. Rubakov for useful discussions. This work has been supported in part by Russian Foundation for Basic Research, grant 02-02-17398, and the grant of the President of Russian Federation NS-2184.2003.2. The work of S.S. has been

supported in part by INTAS grant YS 2001-2/141. S.S. is grateful to DESY Theory Group in Hamburg and Institute of Theoretical Physics, EPFL, Lausanne for hospitality during his visits to these organizations.

\section{References}

[1] D. J. Gross and N. A. Nekrasov, JHEP 0103, 044 (2001) [arXiv:hep-th/0010090].

[2] D. Bak, K. M. Lee and J. H. Park, Phys. Lett. B 501, 305 (2001) [arXiv:hep-th/0011244].

[3] D. Bak and K. M. Lee, Phys. Lett. B 509, 168 (2001) [arXiv:hep-th/0103148].

[4] S. V. Demidov, S. L. Dubovsky, V. A. Rubakov and S. M. Sibiryakov, "Gauge theory solitons on noncommutative cylinder," arXiv:hep-th/0301047, to appear in Theor. Math. Phys.

[5] N. Seiberg and E. Witten, JHEP 9909, 032 (1999) [arXiv:hep-th/9908142].

[6] S. L. Dubovsky and S. M. Sibiryakov, Nucl. Phys. B 664, 407 (2003) [arXiv:hepth/0303233].

[7] D. Bak and S. W. Kim, Nucl. Phys. B 622, 95 (2002) [arXiv:hep-th/0108207].

[8] D. Bak and A. Karch, Nucl. Phys. B 626, 165 (2002) [arXiv:hep-th/0110039].

[9] D. Bak and N. Ohta, Phys. Lett. B 527, 131 (2002) [arXiv:hep-th/0112034].

[10] D. Bak, N. Ohta and M. M. Sheikh-Jabbari, JHEP 0209, 048 (2002) [arXiv:hepth/0205265]. 
[11] T. Banks, W. Fischler, S. H. Shenker and L. Susskind, Phys. Rev. D 55, 5112 (1997) [arXiv:hep-th/9610043].

[12] J. A. Harvey, "Komaba lectures on noncommutative solitons and D-branes," arXiv:hepth/0102076.

M. R. Douglas and N. A. Nekrasov, Rev. Mod. Phys. 73, 977 (2001) [arXiv:hepth/0106048].

A. Konechny and A. Schwarz, Phys. Rept. 360, 353 (2002) [arXiv:hep-th/0012145, hep-th/0107251].

[13] M. M. Sheikh-Jabbari, Phys. Lett. B 455, 129 (1999) [arXiv:hep-th/9901080].

[14] D. Bigatti and L. Susskind, Phys. Rev. D 62, 066004 (2000) [arXiv:hep-th/9908056].

[15] W. I. Taylor, "The M(atrix) model of M-theory," arXiv:hep-th/0002016.

[16] M. R. Douglas, Nucl. Phys. Proc. Suppl. 68, 381 (1998) [arXiv:hep-th/9707228].

[17] W. I. Taylor and M. Van Raamsdonk, Nucl. Phys. B 558, 63 (1999) [arXiv:hepth/9904095].

[18] C. M. Hull, Phys. Lett. B 139, 39 (1984).

[19] D. Berenstein, J. M. Maldacena and H. Nastase, JHEP 0204, 013 (2002) [arXiv:hepth/0202021].

[20] J. G. Russo and A. A. Tseytlin, JHEP 0209, 035 (2002) [arXiv:hep-th/0208114].

[21] G. Bonelli, Nucl. Phys. B 649, 130 (2003) [arXiv:hep-th/0209225].

[22] T.D. Lee and C.N. Yang, Phys. Rev. 104, 254 (1956);

I.Yu. Kobzarev, L.B. Okun and I.Ya. Pomeranchuk, Yad. Fiz. 3, 1154 (1966);

M. Pavšič, Int. J. Theor. Phys. 9, 229 (1974);

S. Blinnikov and M. Khlopov, Sov. Astron. 27, 371 (1983).

[23] Z. Berezhiani, arXiv:hep-ph/0312335.

[24] N. Arkani-Hamed, S. Dimopoulos, G. R. Dvali and N. Kaloper, JHEP 0012, 010 (2000) [arXiv:hep-ph/9911386]. 
[25] F. Dyson, "Looking for life in unlikely places.", Pomeranchuk Prize Lecture, ITEP, Moscow, October 2003 